\title{
Synthesis of $\mathrm{GeSe}_{2}$ Nanobelts Using Thermal Evaporation and Their Photoelectrical Properties
}

\author{
Lijie Zhang, ${ }^{1,2}$ Hongfei Yu, ${ }^{2}$ Yun Yang, ${ }^{2}$ Keqin Yang, ${ }^{2}$ Youqing Dong, ${ }^{2}$ Shaoming Huang, \\ Ning Dai, ${ }^{1}$ and Da-Ming $\mathrm{Zhu}^{1,3}$ \\ ${ }^{1}$ National Laboratory for Infrared Physics, Shanghai Institute of Technical Physics, Chinese Academy of Sciences, \\ Shanghai 200083, China \\ ${ }^{2}$ Nanomaterials and Chemistry Key Laboratory, Wenzhou University, Wenzhou 325027, China \\ ${ }^{3}$ University of Missouri-Kansas City, Kansas City, MO 64110, USA \\ Correspondence should be addressed to Da-Ming Zhu; zhud@umkc.edu
}

Received 12 March 2014; Accepted 14 April 2014; Published 17 June 2014

Academic Editor: Li Li

Copyright (C) 2014 Lijie Zhang et al. This is an open access article distributed under the Creative Commons Attribution License, which permits unrestricted use, distribution, and reproduction in any medium, provided the original work is properly cited.

\begin{abstract}
$\mathrm{GeSe}_{2}$ nanobelts were synthesized via a simple thermal-evaporation process by using gold particles as catalyst and $\mathrm{GeSe}_{2}$ flakes as starting materials. The morphology, crystal structure, and composition were characterized with scanning electron microscopy (SEM), high-resolution transmission electron microscopy (TEM), X-ray diffraction spectroscopy (XRD), X-ray photoelectron spectroscopy (XPS), and energy-dispersive X-ray spectroscopy (EDS). SEM micrographs show that most of GeSe ${ }_{2}$ nanobelts have distinct segmented structures (wide belt, zigzag belt, and narrow belt). A possible mechanism was proposed for the growth of segmented nanobelts. It is possible that the growth of the segmented nanobelts is dominated by both vapor-liquid-solid and vapor-solid mechanisms. Devices made of single $\mathrm{GeSe}_{2}$ nanobelt have been fabricated and their photoelectrical property has been investigated. Results indicate that these nanobelt devices are potential building blocks for optoelectronic applications.
\end{abstract}

\section{Introduction}

Recently, one-dimensional semiconductor nanostructures, such as nanowires and nanobelts or nanoribbons, have attracted much attention because of their excellent physical properties and their unique structure for device applications [1]. They are considered to be excellent building blocks for devices such as field effect transistors [2], field emitters [35], photodetectors [6-8], solar cells [9-11], nanolasers [12], and chemical [13] and biological sensors [14]. These devices demonstrate improved features in comparison to those using bulk materials. For instance, photodetectors fabricated using nanowire or nanobelt always exhibit high sensitivity to light because of their high ratio of surface to volume [8].

IV-VI chalcogenides with layered crystal structure have been widely employed in construction of devices used in optics, thermoelectrics, and optoelectronics [15-17]. As an important member of IV-VI semiconductors, $\mathrm{GeSe}_{2}$ with a wide band gap $(\sim 2.7 \mathrm{eV})$ was found to have promising applications in electronics, optoelectronics, and renewable energy devices [18]. Therefore, much attention has been drawn towards the preparation and the study of property of $\mathrm{GeSe}_{2}$ nanostructures (nanowires, nanobelts, etc.). For instance, three-dimensional $\mathrm{GeSe}_{2}$ nanostructures composed of nanobelts were synthesized with chemical vapor deposition (CVD) and have demonstrated very impressive performance in supercapacitor applications $[15,18]$. $\mathrm{GeSe}_{2}$ nanowires also could be synthesized through decomposing organic ammonium precursor [19]. Stepped-surfaced $\mathrm{GeSe}_{2}$ nanobelts were obtained via CVD process and showed high photoresponsivity and gain [20]. Single $\mathrm{GeSe}_{2}$ nanobelt twoterminal device exhibited high electronic transport properties, photoconductive characteristics, and temperaturedependent electronic characteristics [21].

In most cases, one-dimensional $\mathrm{GeSe}_{2}$ nanostructures were synthesized via a wet-chemical routine or CVD process 


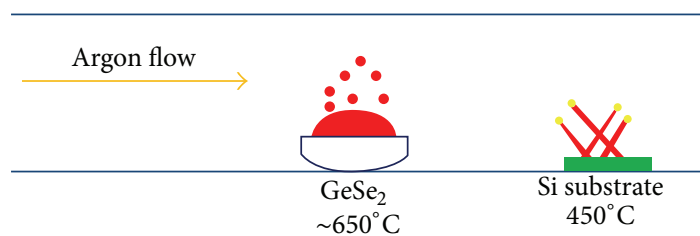

FIGURE 1: Schematic diagram of the synthesis of $\mathrm{GeSe}_{2}$ nanobelts.

by using Ge and Se powders as starting materials $[15,18-$ 20]. It is also known that both GeSe and $\mathrm{GeSe}_{2}$ have high stability $[17,18]$. As a result, both $\mathrm{GeSe}$ and $\mathrm{GeSe}_{2}$ phases probably form simultaneously in the CVD process when Se and $\mathrm{Ge}$ are used as precursors. Besides, the melting point of $\mathrm{Ge}\left(938.25^{\circ} \mathrm{C}\right)$ is much higher than that of Se $\left(217^{\circ} \mathrm{C}\right)$, resulting in different evaporation rate of the precursors and a complex process of the growth.

We synthesized $\mathrm{GeSe}_{2}$ nanobelts via a simple thermal evaporation method by using $\mathrm{GeSe}_{2}$ as starting materials and gold films as catalysts. XRD and TEM results demonstrate that the products are pure phase $\mathrm{GeSe}_{2}$. Besides, vapor-solid (VS) mechanism might contribute to the formation of $\mathrm{GeSe}_{2}$ nanobelts. Devices made of a single $\mathrm{GeSe}_{2}$ nanobelt were fabricated and their photoelectrical property was investigated. Results indicate that these prepared nanobelts have good photoelectrical properties and potential application in optoelectronic devices. The detailed results and the discussion about the synthesized nanobelts and their photoelectrical properties are given in the following sections.

\section{Experimental Section}

The experimental setup used for nanobelt synthesis consists of a horizontal tube furnace, a quartz tube, a gas supply, and a control system (Figure 1). Commercial GeSe $e_{2}$ flakes (20 mg, purity $99.99 \%$, J\&K Scientific Ltd.) used as source materials were positioned in the center of the furnace. The Au-coated $(\sim 20 \mathrm{~nm})$ Si substrates were placed at the downstream zone to collect products. After the furnace was fully flushed with high-purity argon gas for $30 \mathrm{~min}$, the system was heated up to $650^{\circ} \mathrm{C}$ at a rate of $30^{\circ} \mathrm{C} / \mathrm{min}$. The Ar flux was kept at 100 standard cubic centimeters per minute $(\mathrm{sccm})$ and the deposition temperature was $\sim 450^{\circ} \mathrm{C}$. After $1 \mathrm{~h}$ of growth, the system was cooled to room temperature. The yellow products on the substrates were $\mathrm{GeSe}_{2}$ nanobelts.

The morphology, crystal structure, and composition of as-synthesized products were characterized using SEM (FEI Nova NanoSEM200), XRD (Bruker D8 Advanced X-ray diffractometer, $\mathrm{Cu} \mathrm{K} \alpha$ radiation with $\lambda=0.15418 \mathrm{~nm}$ ), TEM (JEOL 2100F, $200 \mathrm{kV}$ ), and EDS. The binding energy of the samples was examined by XPS (Kratos AXIS Ultra DLD).

To evaluate the photoelectrical properties of the nanobelts, two-terminal device made of a single nanobelt was fabricated. The as-synthesized nanobelts were dispersed on $\mathrm{p}^{+}-\mathrm{Si}$ wafer with marks and then treated with electron beam lithography $(30 \mathrm{kV}, 110 \mathrm{pA}$, Nanometer Pattern Generation System installed in the SEM), metallization, and lift-off process to define the $\mathrm{Cr}(10 \mathrm{~nm}) / \mathrm{Au}(100 \mathrm{~nm})$ contacts with the nanobelt. The electrodes were used for interconnecting and the marks were used for aligning in the following electron beam lithography. The current-voltage $(I-V)$ characteristics of the device were investigated in air and at room temperature with a semiconductor characterization system (4200 SCS, Keithley Instruments Inc., USA). The power density of the incident light was calibrated by using a standard silicon photodetectors.

\section{Results and Discussion}

Figure 2 shows the SEM images of the as-prepared nanobelts. As can be seen from the SEM images, most of the nanobelts have segmented $\mathrm{A}-\mathrm{B}-\mathrm{C}$ structure $(\mathrm{A}=$ wide belt, $\mathrm{B}=$ zigzag belt, and $\mathrm{C}=$ narrow belt). The length of the A-B-C structured nanobelts varies from hundreds of micrometers to millimeters (Figures 2(a) and 2(b)). Sections A and C are commonly seen belt-like structure (Figures 2(c)-2(e)) . The thickness of most segmented nanobelts ranged from 60 to $200 \mathrm{~nm}$ (Figure 2(e)). Besides, section C is narrower than section A. In Figure 2(d), particle (catalyst) can be clearly observed due to contrast difference, indicating that VLS mechanism probably dominates the growth of the AB-C structured nanobelts. Interestingly, section B has zigzag structure. Figure 2(f) clearly reveals that the zigzag structure (indicated by arrows) serves as a transition region connecting sections A and C. Figures 2(g) and 2(h) show the enlarged views of section $B$ and the zigzag structure is clearly observed. It is well known that there is a good correlation between the nanobelt diameter and the catalyst size during VLS growth [22]. Although the size of section $\mathrm{C}$ and particle on the tip match well, section B has different shape and size. Therefore, VLS mechanism only partly makes contribution to the growth of nanobelt possibly. As can be seen from Figures $2(\mathrm{~g})$ and $2(\mathrm{~h})$, the growth of flake-like branch on the backbone might be governed by vapor solid (VS) rather than VLS mechanism. Besides, some small branches were also observed possibly due to different growth stages. As a layer-structured chalcogenides, $\mathrm{GeSe}_{2}$ consists of Se-Ge-Se layers stacked together via van der Waals interaction [20]. The branches with different thickness are found (e.g., indicated by arrows 3 and 4 in Figure 2(h)), implying that each layer has different growth speed. The segmented nanobelts have uniform thickness at section A. Besides, the growth direction is from section $\mathrm{A}$ to $\mathrm{C}$ according to the top growth mechanism. Therefore, it is reasonable to conclude that the zigzag structures are not an ultimate state and they would grow into nanobelt with the same structure as section $\mathrm{A}$ if growth continues.

XPS was employed to derive composition information of the as-synthesized nanobelts. The binding energy of all the survey and finely scanned spectrums was calibrated by the standard reference of the $\mathrm{C} 1 \mathrm{~s}(284.8 \mathrm{eV})$ signal. Typical XPS survey spectrum (Figure 3(a)) indicates the presence of Ge and Se. Figure 3(b) shows that the peaks of Se 3d3/2 and Se $3 \mathrm{~d} 5 / 2$ core level are at 55.1 and $54.4 \mathrm{eV}$, respectively. The peak position of Ge $3 \mathrm{~d}$ core level is at $31.1 \mathrm{eV}$ which is consistent with the literature [20]. The Ge $3 \mathrm{~d}$ core level 


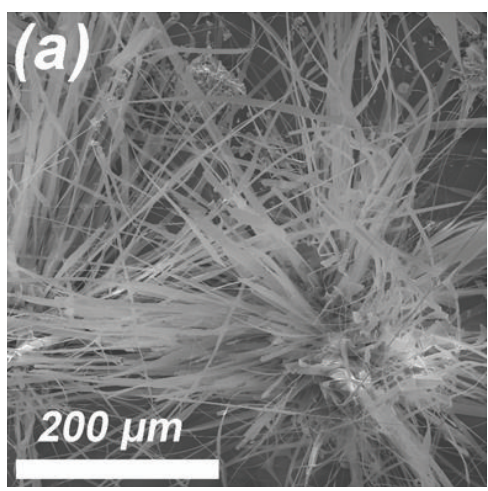

(a)

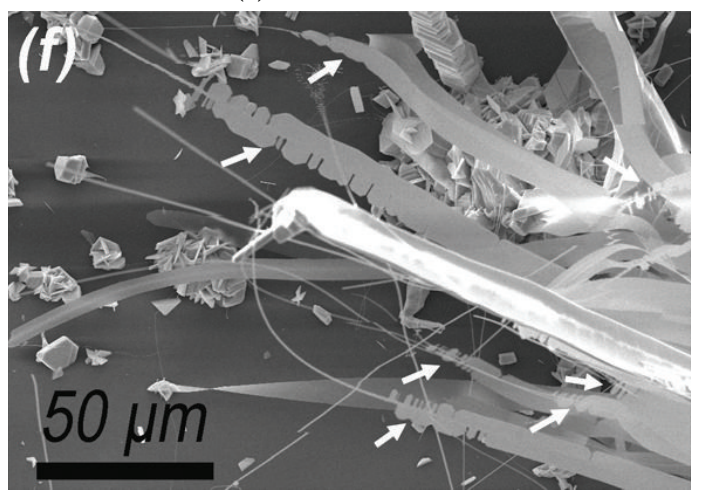

(f)

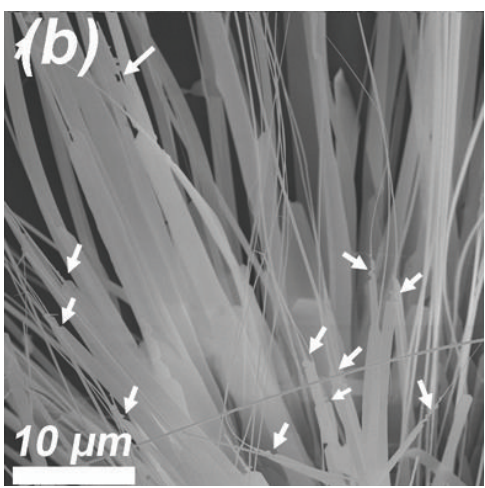

(b)

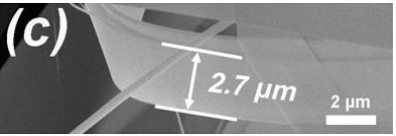

(c)

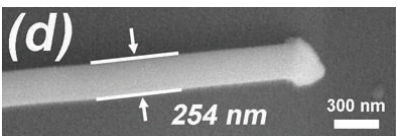

(d)

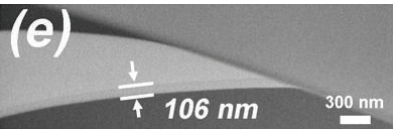

(e)

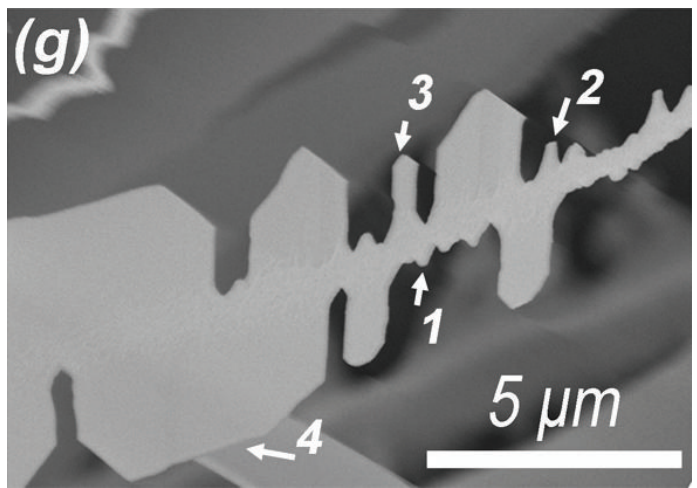

(g)

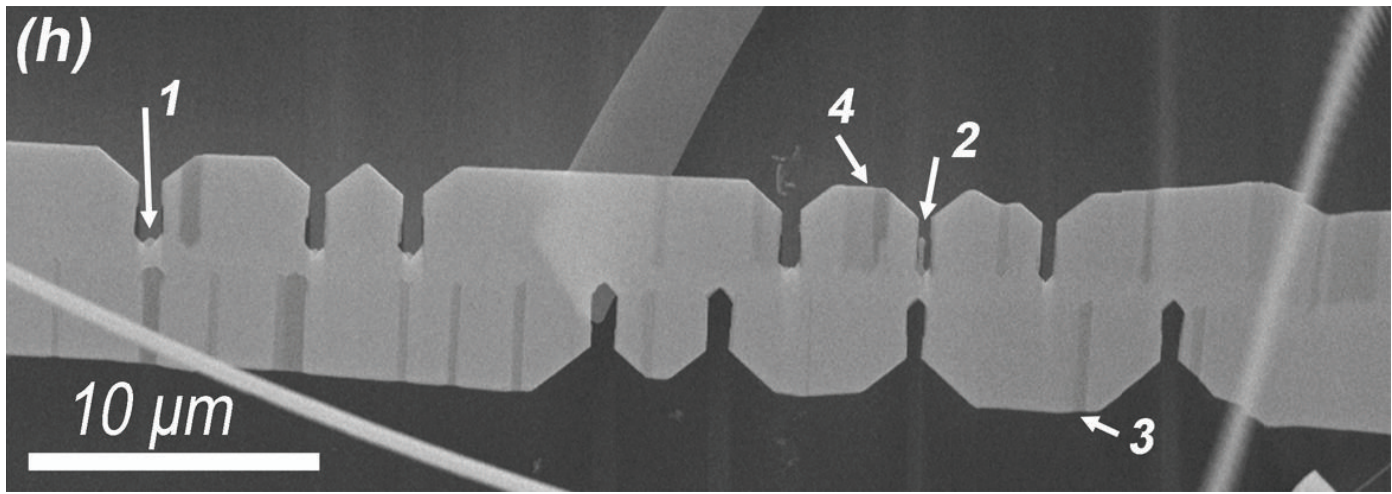

(h)

Figure 2: SEM images of the segmented nanobelts with A-B-C structure ( $\mathrm{A}=$ wide belt, $\mathrm{B}=$ zizag belt, and $\mathrm{C}=$ narrow belt). (a) A low magnification image of the segmented nanobelts. (b) An enlarged view. (c-e) SEM images taken from section A, section B, and side of section $\mathrm{A}$, respectively. ( $\mathrm{f}-\mathrm{h})$ SEM images taken from section $\mathrm{B}$.

spectrum is asymmetric and a small shoulder on the high energy side is found (Figure 3(c)). This is caused by the Ge$\mathrm{O}$ states (corresponding to $33 \mathrm{eV}$ ) on the surfaces [23]. No signals from $\mathrm{Ge}-\mathrm{Ge}(29.1 \mathrm{eV})$ and $\mathrm{Se}-\mathrm{Se}(55.4 \mathrm{eV})$ [20] were detected in the as-synthesized nanobelts, demonstrating that the products are pure phase $\mathrm{GeSe}_{2}$.

Figure 4(a) shows a typical XRD pattern of the assynthesized nanobelts. All the diffraction peaks can be indexed to single-crystalline monoclinic $\mathrm{GeSe}_{2}$ (JCPDS file: 71-0117). A representative TEM image (Figure 4(b)) further confirms the belt-like morphology. Figure 4(c) shows the high-resolution TEM (HRTEM) image of the nanobelt edge, confirming that the nanobelt is a single crystal and defectfree. Two d-spacings of 1.68 and $0.70 \mathrm{~nm}$ match well with the (010) and (100) planes of monoclinic $\mathrm{GeSe}_{2}$, respectively. The reciprocal lattice peaks (Figure $4(\mathrm{~d})$ ) which were given by fast Fourier transform (FFT) can be indexed to a monoclinic structure of $\mathrm{GeSe}_{2}$. The indexed FFT pattern demonstrates that the growth direction of nanobelt is along [010] direction, which is inconsistent with the [112] growth direction of stepped-surfaced $\mathrm{GeSe}_{2}$ nanobelts synthesized via VLS mode [20]. The top and bottom surfaces of the nanobelts are $\{001\}$ facets, and the side surfaces are $\{100\}$ facets.

Figure 4(e) shows the scanning transmission electron microscope (STEM) image of a $\mathrm{GeSe}_{2}$ nanobelt with catalyst droplet on the tip. Figures $4(\mathrm{f})-4(\mathrm{~h})$ show elemental distribution of $\mathrm{Au}, \mathrm{Ge}$, and Se, respectively. Au was detected only at the catalyst droplet, whereas Ge and Se were observed in the 


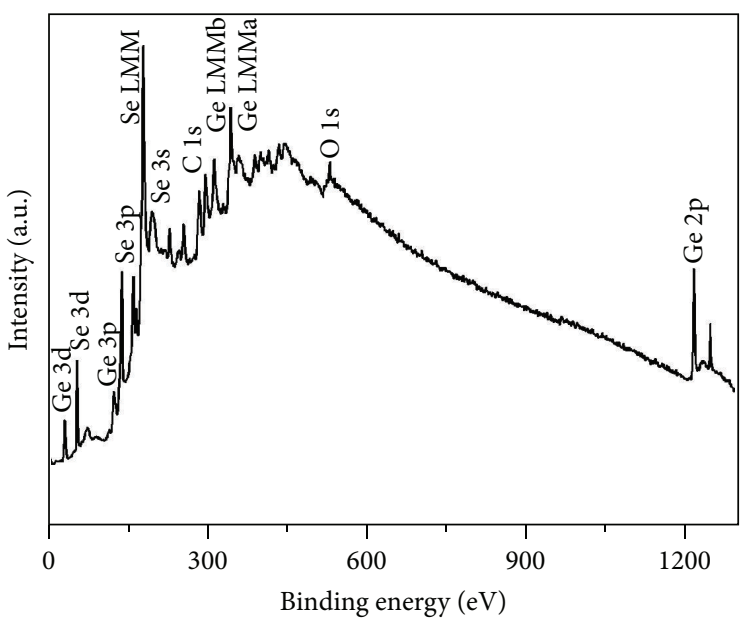

(a)

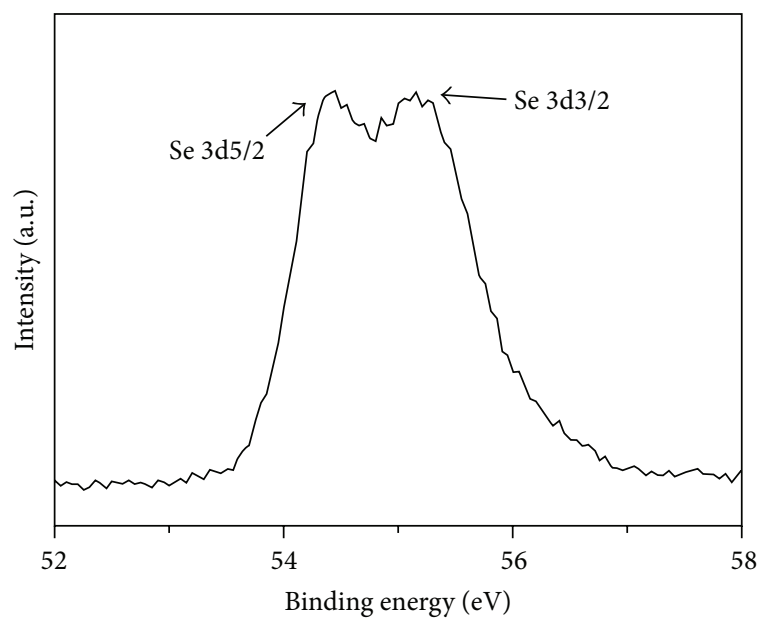

(b)

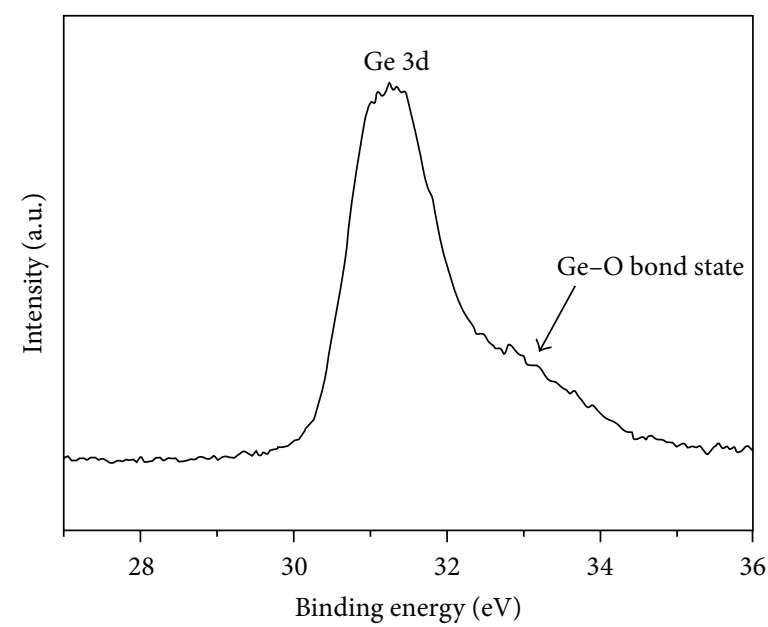

(c)

FIGURE 3: XPS analysis of the nanobelts. (a) XPS survey spectrum of the as-synthesized nanobelts. (b) High-resolution XPS spectrum of Se $3 \mathrm{~d} 3 / 2$ and $3 \mathrm{~d} 5 / 2$ core level. (c) High-resolution XPS spectrum of Ge $3 \mathrm{~d}$ and G-O bond state.

nanobelt as well as the catalyst droplet. This further confirms the VLS growth of the nanobelts. Under high temperature, Au film changes into liquid droplet. Meanwhile, high temperature causes the formation of $\mathrm{GeSe}_{2}$ vapor which enters the $\mathrm{Au}$ particles to produce $\mathrm{Au}-\mathrm{Ge}-\mathrm{Se}$ alloy particles. When $\mathrm{GeSe}_{2}$ is oversaturated, it recrystallizes out of alloy particles, nucleates, and further grows to nanobelt. Figure 4(i) is the STEM image of single nanobelt. Figures $4(\mathrm{j})$ and $4(\mathrm{k})$ display EDS profiles along the red and green arrows in Figure 4(i), respectively. The STEM-EDS results confirm the uniform distribution of $\mathrm{Ge}$ and Se in the nanobelt. The atomic ratio between Se and Ge is close to stoichiometric ratio $(2: 1)$.

HRTEM was employed to investigate the structure of section B. Figure 5(a) shows a representative TEM image of a zigzag structure. Figure 5(b) is the HRTEM image of selected region marked by rectangle 1 in Figure 5(a) and the inset shows the corresponding FFT pattern. It can be found that the crystal structure of branch is identical to that of the backbone, further confirming that the growth of branches is from the backbone. Figures 5(c)-5(f), respectively, show the HRTEM images of position marked by rectangles 2-5 in Figure 5(a). Figure 5(g) gives an enlarged view of the region marked by rectangular area in Figure 5(f) and the corresponding FFT pattern is shown in Figure 5(h). These results indicate that the $\mathrm{GeSe}_{2}$ nanobelt grows along [010] direction. The VS growth direction of section $\mathrm{B}$ is along [100] and [-100] directions. The layered structure of the $\mathrm{GeSe}_{2}$ is clearly observed in Figures 5(b) and 5(f), which is consistent with previous report [15] that Se-Ge-Se layers are assembled along [001] direction. As a result, the VS growth is parallel to (001) plane rather than perpendicular to (001). The HRTEM results also suggest that the VS growth speed of each layer is not equal and this result is in agreement with the SEM observation (Figure 2(h)). With the VS growth continuing, the side surfaces are changed from $\{-110\}$ and $\{-1-10\}$ facets (Figures $5(\mathrm{f})$ and $5(\mathrm{~g}))$ to $\{-120\}$, $\{-1-20\}$, and $\{010\}$ facets (Figures 5(c) and 5(d)).

Based on the above analysis, a possible growth mechanism is proposed for the formation of the segmented nanobelts. Firstly, Au-Ge-Se alloy particles act as catalyst and induce the growth along [010] direction via VLS mode 


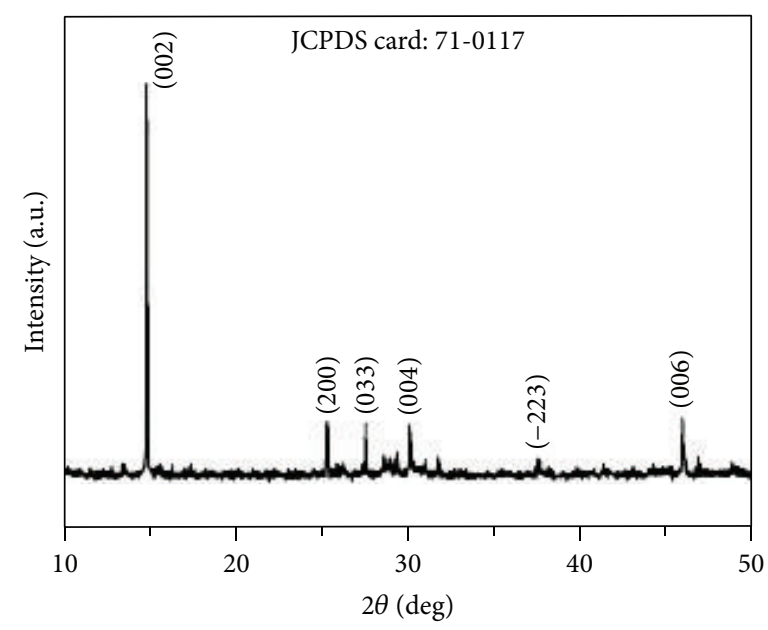

(a)

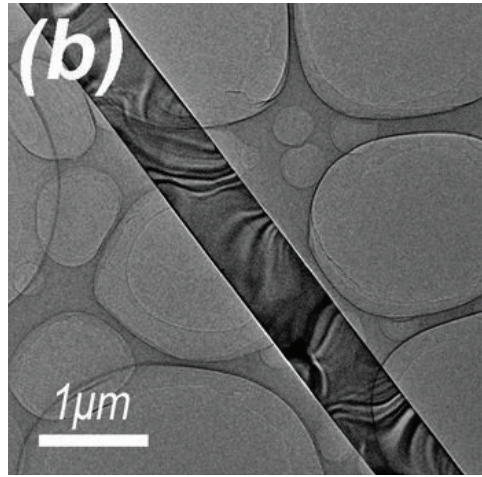

(b)

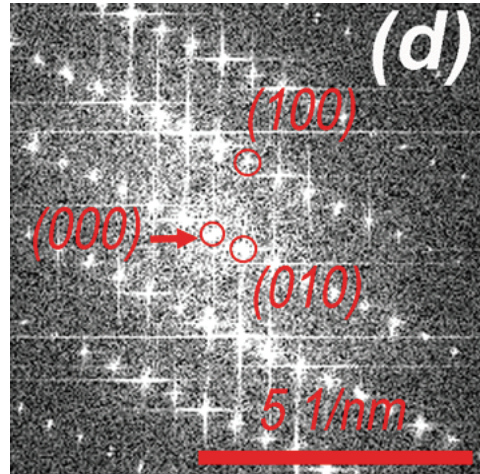

(d)

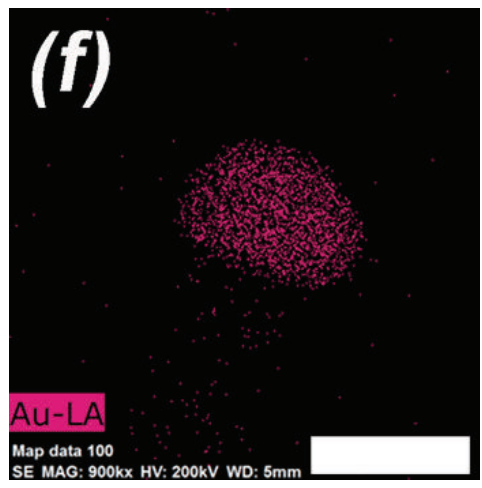

(f)

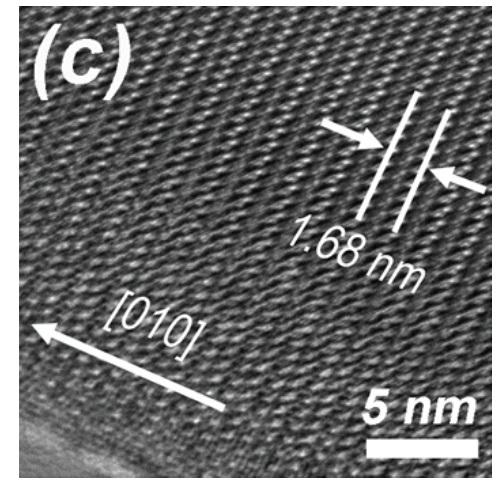

(c)

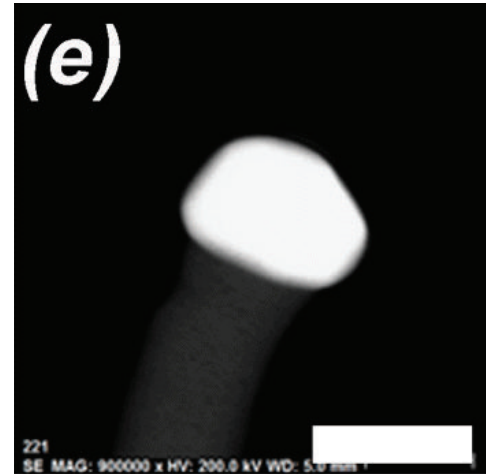

(e)

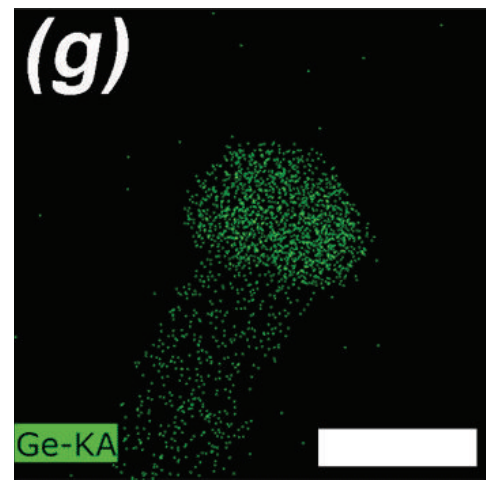

(g)

FIgURE 4: Continued. 


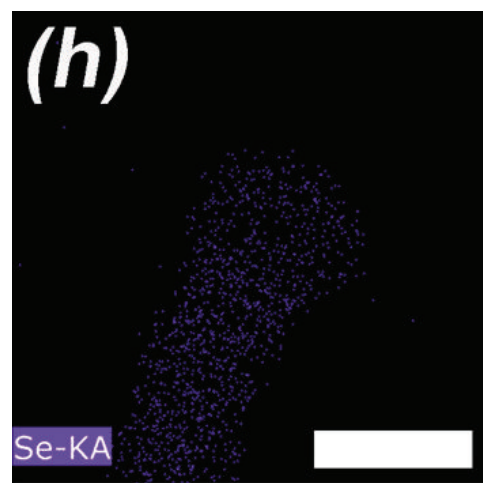

(h)

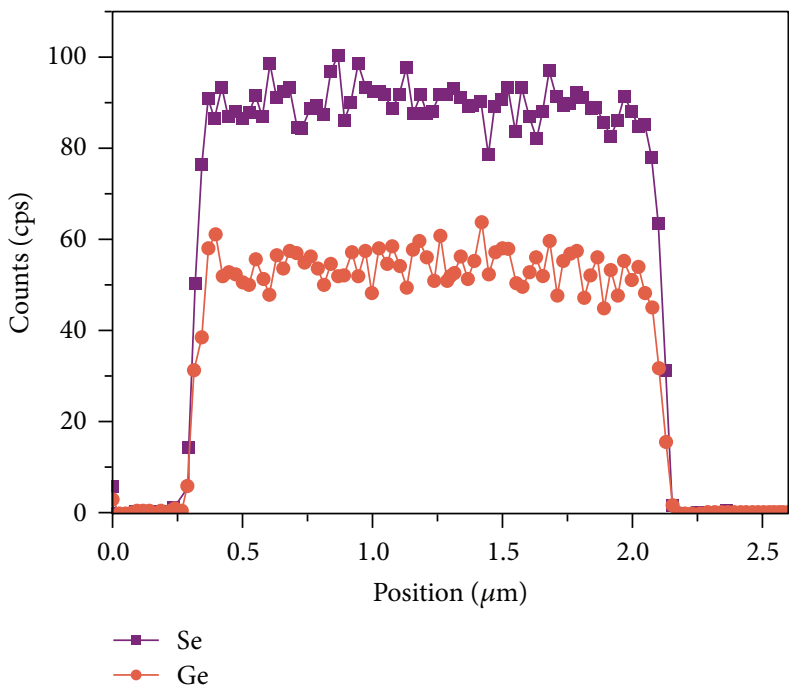

(j)

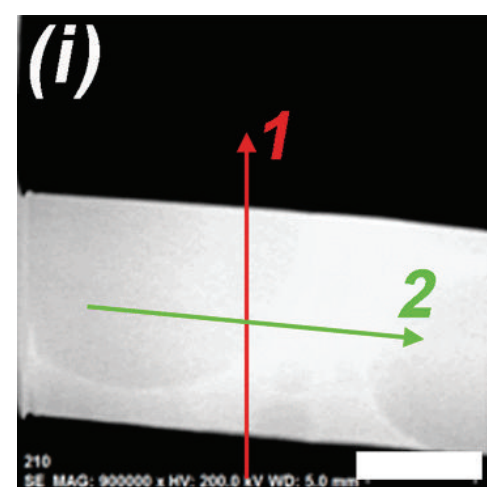

(i)

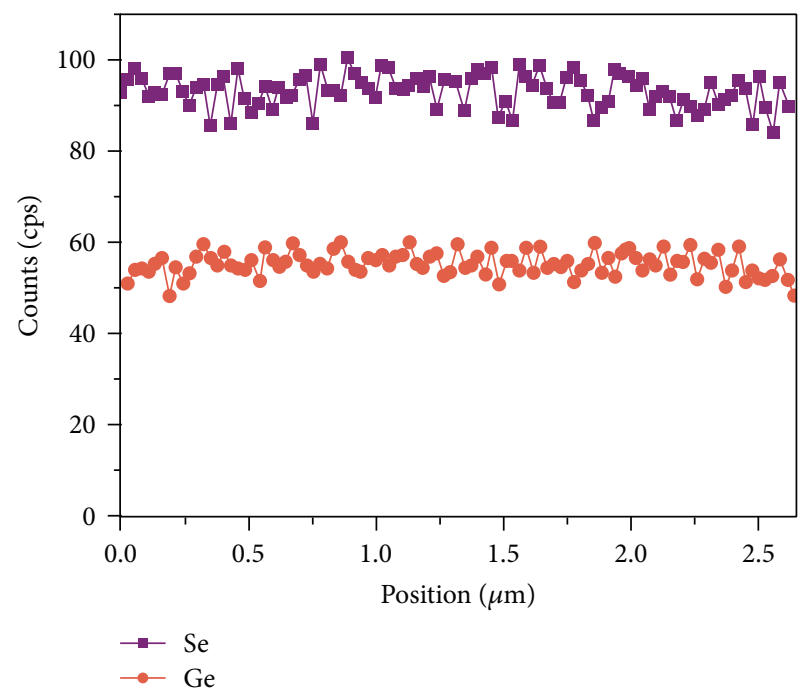

$(\mathrm{k})$

FIGURE 4: (a) XRD pattern of the nanobelts. (b) A TEM image of a nanobelt. (c, d) A HRTEM taken from the edge of the nanobelt and corresponding FFT pattern. (e) A STEM image taken from the tip of a nanobelt. (f-h) EDS maps for Au, Gen and Se, respectively. Scale bar: $100 \mathrm{~nm}$. (i) A STEM image of the nanobelt. Scale bar: $1 \mu \mathrm{m}$. (j, k) EDS profiles taken along the red and green arrows in (i), respectively.

(Figure 6(a)). The two side surfaces of a nanobelt in this growth stage are parallel to (100) plane. Once the narrow nanobelt is formed, its side surfaces become nucleation sites which direct the VS growth along [100] and [-100] directions (Figure 6(b)). However, because the growth speed of the VLS mode is faster than that of VS mode, zigzag structure forms (Figure 6(b)). With the VLS and VS growth proceeding simultaneously, the zigzag product evolutes to nanobelt with the structure of section A and the length of the section C further increase (Figure 6(c)), resulting in the formation of A-B-C structured products.

Compared with bulk materials, nanostructured semiconductors have large surface-to-volume ratio and dangling bonds on the material surfaces. Therefore, there are numerous oxygen molecules absorbed on their surface which attract the electron of photoexcited carriers $\left(\mathrm{O}^{2}+\mathrm{e}^{-} \rightarrow \mathrm{O}_{2}^{-}\right)$. This can improve the separation of photoexcited carriers and increase photocurrent [24]. To evaluate the photoelectrical properties of the as-synthesized nanobelts, two-terminal device was fabricated through using a single $\mathrm{GeSe}_{2}$ nanobelt.
Figure 7(a) shows the schematic illustration of experimental setup for photoelectrical measurements. A white light with power density of $\sim 0.7 \mathrm{~mW} / \mathrm{mm}^{2}$ was used as the incident light. Figure 7(b) gives the current-voltage $(I-V)$ characteristics of the device under incident light illumination or without illumination. The asymmetry and nonlinearity of $I-$ $V$ curves indicate the formation of Schottky contact between the nanobelt and the metal electrodes. Compared with the dark current at a bias of $1 \mathrm{~V}$, the photocurrent increases by $\sim 52$ times. This indicates that the as-synthesized nanobelts have good photoelectrical properties.

\section{Conclusion}

In summary, we demonstrated that a simple thermal evaporation method could be used to synthesize $\mathrm{GeSe}_{2}$ nanobelts through using gold particles as catalyst and $\mathrm{GeSe}_{2}$ flakes as starting materials. Most of nanobelts have wide belt, zigzag belt, or narrow belt structures which are mostly single 


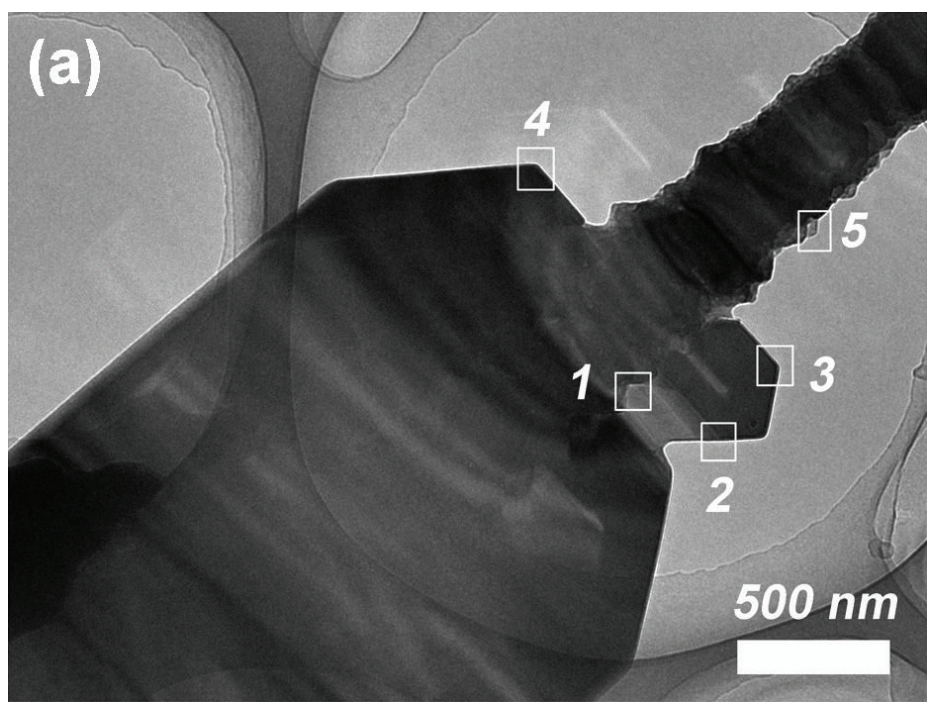

(a)

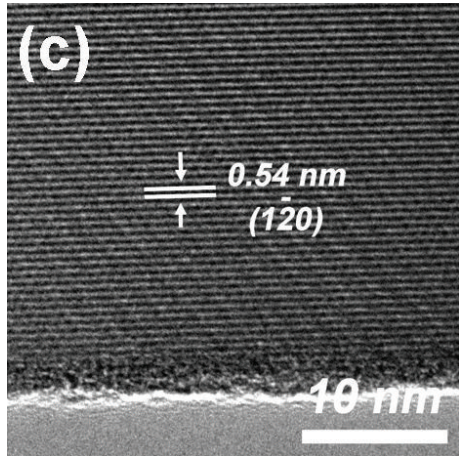

(c)

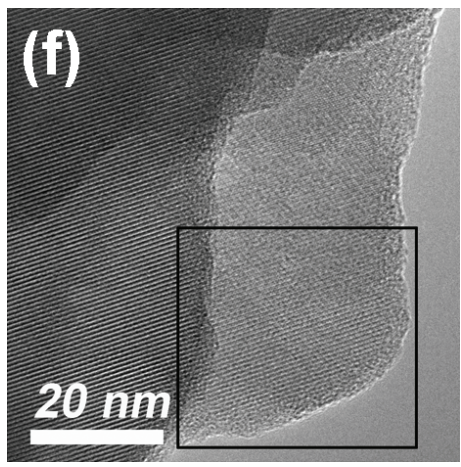

(f)

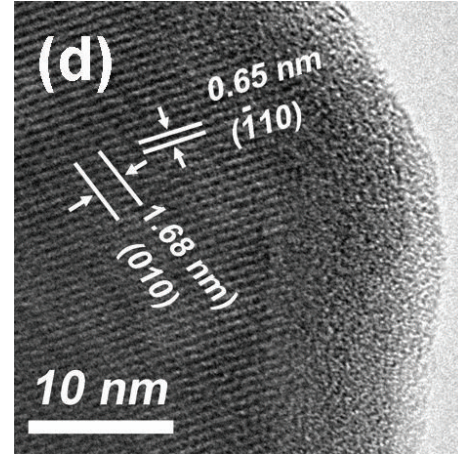

(d)

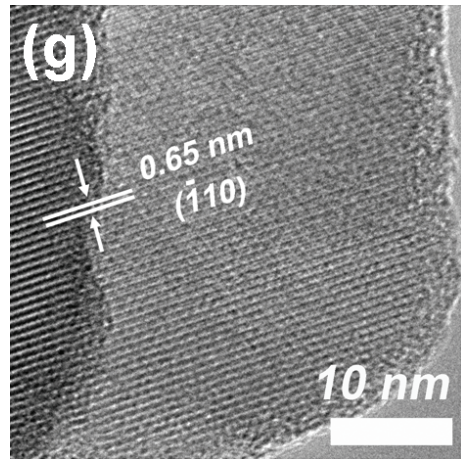

(g)

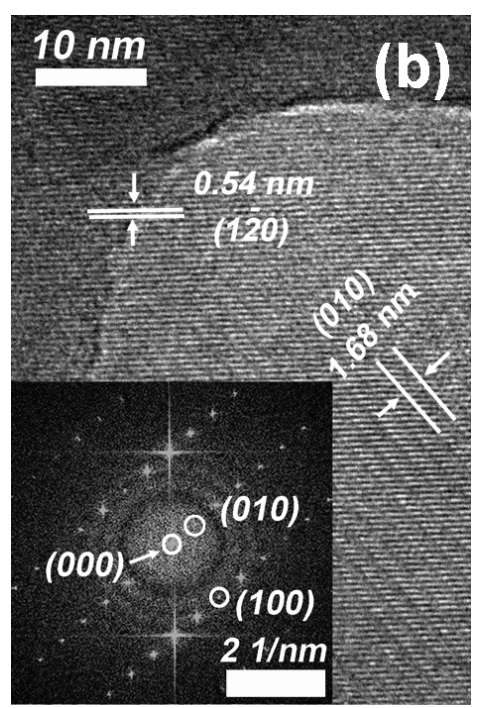

(b)

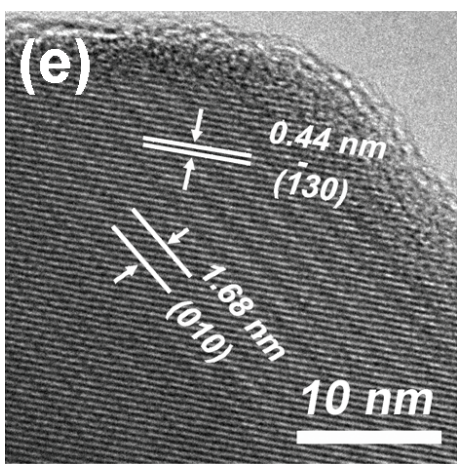

(e)

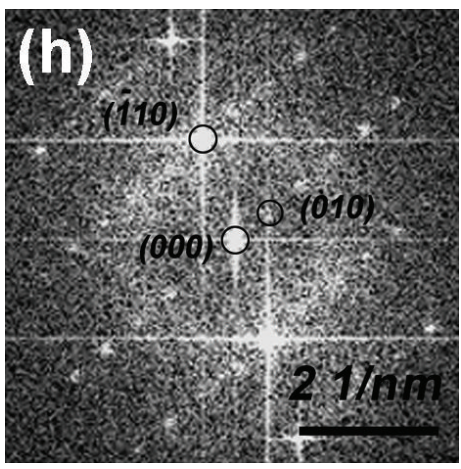

(h)

FIGURE 5: (a) A TEM image of the zigzag structure. (b-f) The corresponding HRTEM taken from the rectangular area 1-5, respectively. The inset in (b) shows its corresponding FFT pattern. $(\mathrm{g}, \mathrm{h})$ Enlarged views of the rectangular area in (f) and corresponding FFT pattern.

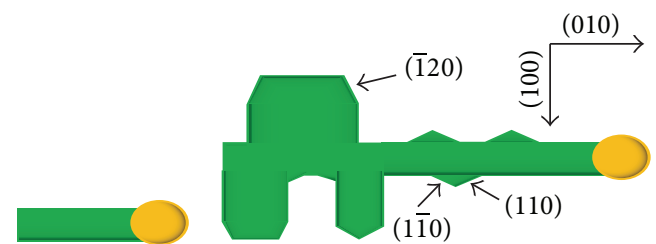

(a)

(b)

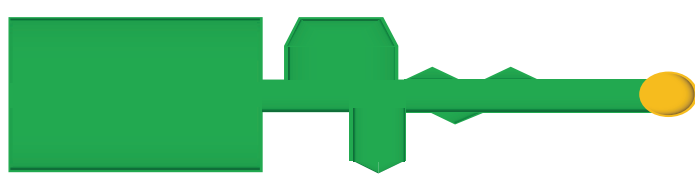

(c)

FIGURE 6: Schematic illustration of the growth process. 


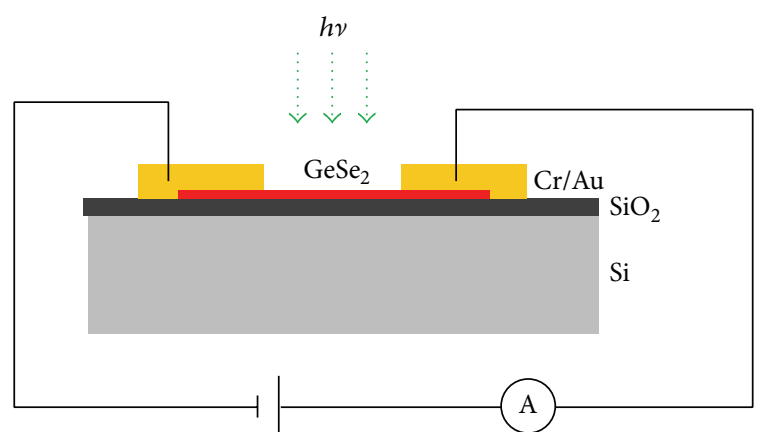

(a)

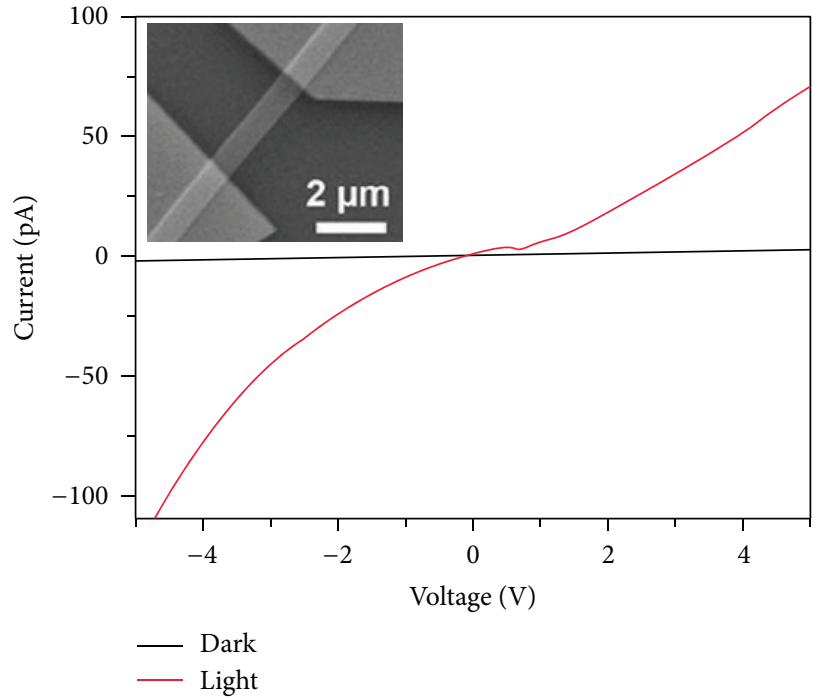

(b)

FiguRE 7: (a) Schematic illustration of the experimental setup for photoelectrical measurements. (b) $I$ - $V$ curves of the two-terminal device under white-light illumination or in dark condition.

crystals. A possible mechanism was proposed for explaining the growth of segmented nanobelts. The growth occurs first along [010] direction via VLS mode and then VS mode starts along [100] and [-100]directions. These two growth modes result in the formation of segmented nanobelts. The assynthesized $\mathrm{GeSe}_{2}$ nanobelts exhibit excellent photoelectrical property.

\section{Conflict of Interests}

The authors declare that there is no conflict of interests regarding the publication of this paper.

\section{Acknowledgments}

This work was supported by NSFC (nos. 61001005 and 11375174), National 973 Project of China (nos. 2012CB934300 and 2012CB619200), and NSFZJ (Y1110734).

\section{References}

[1] W. Wu, X. Wen, and Z. L. Wang, "Taxel-addressable matrix of vertical-nanowire piezotronic transistors for active and adaptive tactile imaging," Science, vol. 340 , no. 6135 , pp. $952-$ 957, 2013.

[2] A. M. Ionescu and H. Riel, "Tunnel field-effect transistors as energy-efficient electronic switches," Nature, vol. 479, no. 7373, pp. 329-337, 2011.

[3] X. Zhang, Y. Chen, Z. Xie, and W. Yang, "Shape and doping enhanced field emission properties of quasialigned 3C-SiC nanowires," Journal of Physical Chemistry C, vol. 114, no. 18, pp. 8251-8255, 2010.

[4] S. Chen, P. Ying, L. Wang et al., "Growth of flexible N-doped SiC quasialigned nanoarrays and their field emission properties,"
Journal of Materials Chemistry C, vol. 1, no. 31, pp. 4779-4784, 2013.

[5] S. Chen, P. Ying, L. Wang et al., "Controlled growth of $\mathrm{SiC}$ flexible field emitters with clear and sharp tips," RSC Advances, vol. 4, no. 16, pp. 8376-8382, 2014.

[6] T. Zhai, L. Li, X. Wang, X. Fang, Y. Bando, and D. Golberg, "Recent developments in one-dimensional inorganic nanostructures for photodetectors," Advanced Functional Materials, vol. 20, no. 24, pp. 4233-4248, 2010.

[7] L. Zhang, H. Yu, W. Cao, C. Zou, Y. Dong, and D. M. Zhu, "Enhanced electrical and optoelectrical properties of cadmium selenide nanobelts by chlorine doping," Micro \& Nano Letters, vol. 9, no. 1, pp. 55-59, 2014.

[8] L. Hu, J. Yan, M. Liao, L. Wu, and X. Fang, "Ultrahigh external quantum efficiency from thin $\mathrm{SnO}_{2}$ nanowire ultraviolet photodetectors," Small, vol. 7, no. 8, pp. 1012-1017, 2011.

[9] Q. Li, L. Zhai, C. Zou et al., "Wurtzite $\mathrm{CuInS}_{2}$ and $\mathrm{CuIn}_{\mathrm{x}} \mathrm{Ga}_{1-\mathrm{x}} \mathrm{S}_{2}$ nanoribbons: synthesis, optical and photoelectrical properties," Nanoscale, vol. 5, no. 4, pp. 1638-1648, 2013.

[10] E. Garnett and P. Yang, "Light trapping in silicon nanowire solar cells," Nano Letters, vol. 10, no. 3, pp. 1082-1087, 2010.

[11] J. Heo, W. Guo, and P. Bhattacharya, "Monolithic single GaN nanowire laser with photonic crystal microcavity on silicon," Applied Physics Letters, vol. 98, no. 2, Article ID 021110, 2011.

[12] D. Cammi and C. Ronning, "Persistent photoconductivity in $\mathrm{ZnO}$ nanowires in different atmospheres," Journal of Nanomaterials, vol. 2014, Article ID 184120, 5 pages, 2014.

[13] X. Li, X. Li, N. Chen et al., "CuO- $\mathrm{In}_{2} \mathrm{O}_{3}$ core-shell nanowire based chemical gas sensors," Journal of Nanomaterials, vol. 2014, Article ID 973156, 7 pages, 2014.

[14] Y. Niu, A. Qin, W. Song et al., "Biocompatible single-crystal selenium nanobelt based nanodevice as a temperature-tunable photosensor," Journal of Nanomaterials, vol. 2012, Article ID 384671, 6 pages, 2012. 
[15] X. Wang, B. Liu, Q. Wang et al., "Three-dimensional hierarchical $\mathrm{GeSe}_{2}$ nanostructures for high performance flexible allsolid-state supercapacitors," Advanced Materials, vol. 25, no. 10, pp. 1479-1486, 2013.

[16] P. D. Antunez, J. J. Buckley, and R. L. Brutchey, “Tin and germanium monochalcogenide IV-VI semiconductor nanocrystals for use in solar cells," Nanoscale, vol. 3, no. 6, pp. 2399-2411, 2011.

[17] B. Mukherjee, Y. Cai, H. R. Tan, Y. P. Feng, E. S. Tok, and C. H. Sow, "NIR Schottky photodetectors based on individual single-crystalline GeSe nanosheet," ACS Applied Materials \& Interfaces, vol. 5, no. 19, pp. 9594-9604, 2013.

[18] Y. Zhang, H. Li, L. Jiang et al., "Field emission from $\mathrm{GeSe}_{2}$ nanowalls," Applied Physics Letters, vol. 98, no. 11, Article ID 113118, 2011.

[19] M. Nath, A. Choudhury, and C. N. R. Rao, "Chemical routes to $\mathrm{GeS}_{2}$ and $\mathrm{GeSe}_{2}$ nanowires," Chemical Communications, no. 23, pp. 2698-2699, 2004.

[20] B. Mukherjee, Z. Hu, M. Zheng et al., "Stepped-surfaced $\mathrm{GeSe}_{2}$ nanobelts with high-gain photoconductivity," Journal of Materials Chemistry, vol. 22, no. 47, pp. 24882-24888, 2012.

[21] B. Mukherjee, E. S. Tok, and C. H. Sow, "Photocurrent characteristics of individual $\mathrm{GeSe}_{2}$ nanobelt with Schottky effects," Journal of Applied Physics, vol. 114, no. 13, Article ID 134302, 2013.

[22] J. Zhu, H. Peng, C. K. Chan, K. Jarausch, X. F. Zhang, and Y. Cui, "Hyperbranched lead selenide nanowire networks," Nano Letters, vol. 7, no. 4, pp. 1095-1099, 2007.

[23] A. Kovalskiy, A. C. Miller, H. Jain, and M. Mitkova, "In situ measurements of X-ray-induced silver diffusion into a $\mathrm{Ge}_{30} \mathrm{Se}_{70}$ thin film," Journal of the American Ceramic Society, vol. 91, no. 3, pp. 760-765, 2008.

[24] J. D. Prades, F. Hernandez-Ramirez, R. Jimenez-Diaz et al., "The effects of electron-hole separation on the photoconductivity of individual metal oxide nanowires," Nanotechnology, vol. 19, no. 46, Article ID 465501, 2008. 

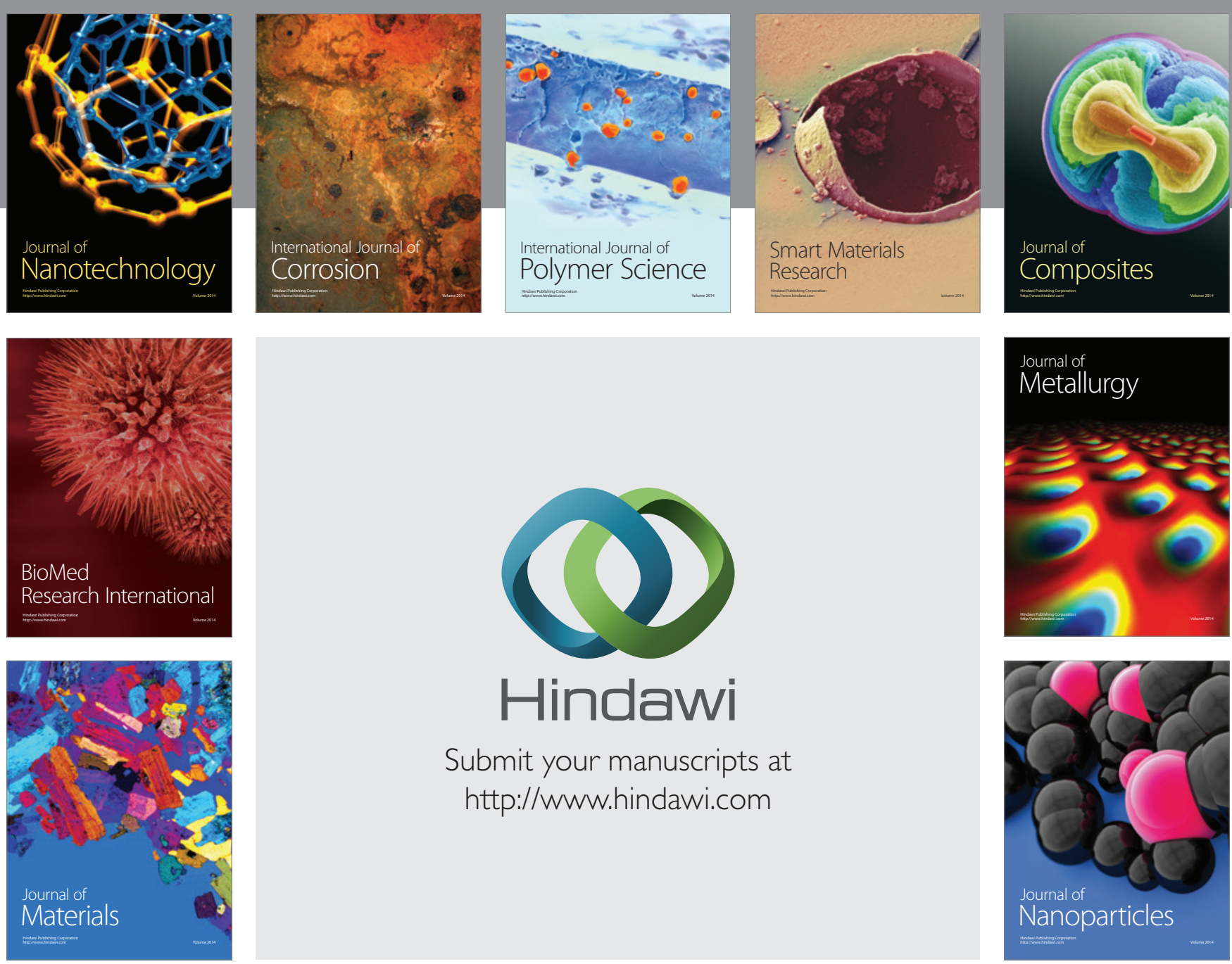

Submit your manuscripts at http://www.hindawi.com
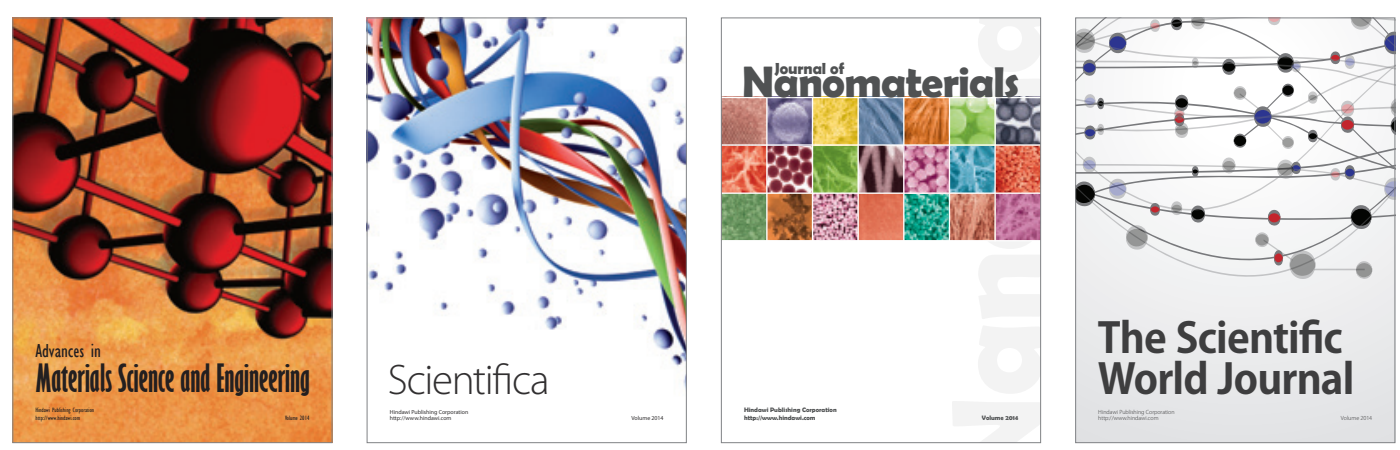

\section{The Scientific World Journal}
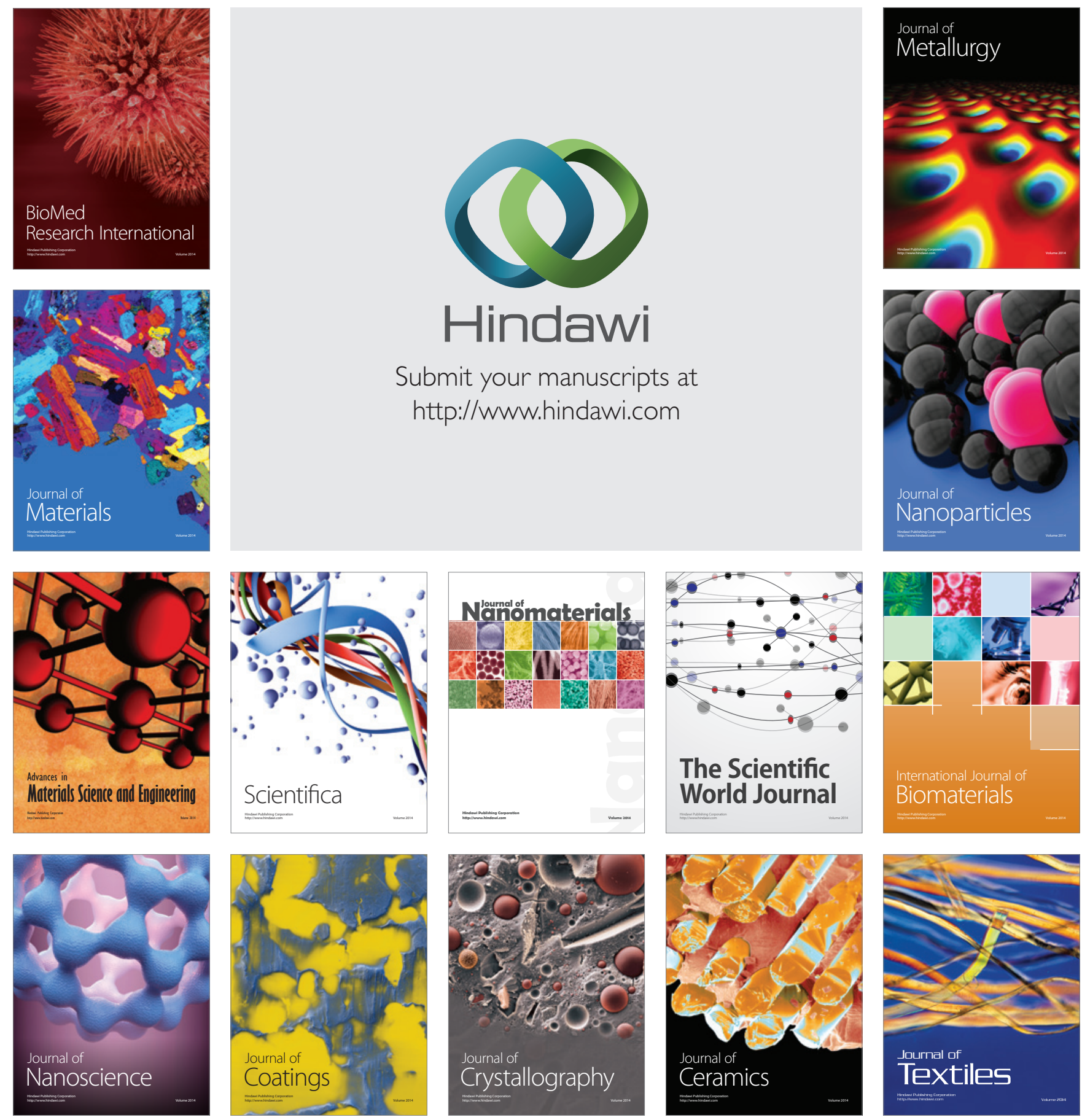